This item was submitted to Loughborough's Research Repository by the author.

Items in Figshare are protected by copyright, with all rights reserved, unless otherwise indicated.

\title{
Dynamic stratification in drying films of colloidal mixtures
}

\section{PLEASE CITE THE PUBLISHED VERSION}

http://dx.doi.org/10.1103/PhysRevLett.116.118301

\section{PUBLISHER}

(C) American Physical Society

\section{VERSION}

AM (Accepted Manuscript)

\section{PUBLISHER STATEMENT}

This work is made available according to the conditions of the Creative Commons Attribution-NonCommercialNoDerivatives 4.0 International (CC BY-NC-ND 4.0) licence. Full details of this licence are available at: https://creativecommons.org/licenses/by-nc-nd/4.0/

\section{LICENCE}

CC BY-NC-ND 4.0

\section{REPOSITORY RECORD}

Fortini, Andrea, Ignacio Martin-Fabiani, Jennifer Lesage de la Haye, Pierre-Yves Dugas, Muriel Lansalot, Franck D'Agosto, Elodie Bourgeat-Lami, Joseph L. Keddie, and Richard P. Sear. 2016. "Dynamic Stratification in Drying Films of Colloidal Mixtures". Loughborough University. https://hdl.handle.net/2134/24875. 


\title{
Dynamic stratification in drying films of colloidal mixtures
}

\author{
Andrea Fortini,,${ }^{1,2, *}$ Ignacio Martín-Fabiani, ${ }^{1}$ Jennifer Lesage De La Haye, ${ }^{3}$ Pierre-Yves Dugas,${ }^{3}$ Muriel \\ Lansalot, ${ }^{3}$ Franck D'Agosto, ${ }^{3}$ Elodie Bourgeat-Lami, ${ }^{3}$ Joseph L. Keddie, ${ }^{1}$ and Richard P. Sear ${ }^{1, \dagger}$ \\ ${ }^{1}$ Department of Physics, University of Surrey, Guildford GU2 7XH, United Kingdom \\ ${ }^{2}$ Theoretische Physik II, Physikalisches Institut, Universität Bayreuth, \\ Universitätsstraße 30, D-95447 Bayreuth, Germany \\ ${ }^{3}$ Laboratoire de Chimie, Catalyse, Polymères et Procédés Université Claude Bernard Lyon 1, France
}

\begin{abstract}
In simulations and experiments, we study the drying of films containing mixtures of large and small colloidal particles in water. During drying, the mixture stratifies into a layer of the larger particles at the bottom with a layer of the smaller particles on top. We developed a model to show that a gradient in osmotic pressure, which develops dynamically during drying, is responsible for the segregation mechanism behind stratification.
\end{abstract}

PACS numbers: 64.75.Xc,68.03.Fg

Solid thin films on surfaces are often made by spreading a thin liquid film containing solid colloidal particles onto the surface, and allowing the liquid to evaporate [1]. As we have known since the time of Robert Brown [2], colloidal particles undergo Brownian motion; they diffuse. As a film dries, the water surface falls, pushing the colloidal particles ahead of it. There is then competition between the particles' Brownian motion and the movement of the surface. The Brownian motion tends to distribute the colloidal particles uniformly in the drying film, while the motion of the surface drives the system out of equilibrium. It is known that this competition determines the distribution of particles on the length-scale of the height $H$ of the film [3-5]. Here we demonstrate a novel self-organization mechanism in colloidal mixtures, which occurs during solvent evaporation. This mechanism separates large and small particles, to form a film stratified by size.

To understand this mechanism, we start by considering the simple case of a film containing only one species of colloidal particle with a diffusion constant $D$. The timescale for diffusion across the height of the film is $H^{2} / D$. During evaporation of the continuous solvent, the top surface moves down with a velocity $v_{e v}$; the evaporation timescale is $H / v_{e v}$. The competition between these two timescales is quantified by the film formation Péclet number $\mathrm{Pe}_{\text {film }}=v_{e v} H / D[1,3]$. The drying film is near equilibrium if $\mathrm{Pe}_{f i l m}<1$, i.e., when the timescale for diffusion is smaller than that for evaporation. In this case, evaporation only weakly perturbs the vertical concentration profile, and the profile remains almost uniform at all times. In the other limit, where $\mathrm{Pe}_{\text {film }}>1$, diffusion cannot keep up with the moving interface, and particles accumulate near the descending interface at the top of the film $[1,3,4,6-10]$. This description applies to one species of colloidal particle.

However, in paints and inks [3], and often in nanofabrication $[11,12]$, there are mixtures of different sizes (and types) of particles. Earlier work has focused on the regime where the film formation Péclet number of the large particles is greater than one, while that of the smaller particles is less than one, and hence large particles form the top layer [5, 13-15]. There stratification is caused by the different rates at which small and large particles accumulate at the falling interface.

In this letter, we show that in the regime where both film Péclet numbers are much larger than one, there is a generic tendency for the small particles to segregate in a layer on top of the larger particles. This novel stratification mechanism is driven by a gradient of osmotic pressure and is found in both computer simulations and experiment on drying films containing mixtures of small and large colloidal particles. This is a previously unknown example of self-organization in a non-equilibrium process.

Moreover, this type of stratification is highly desirable because it allows the independent control of the properties of the top and the bottom of a coating or self-organized nanostructure. The mechanism differs both from equilibrium phase separation and the out-ofequilibrium Brazil-nut effect [16].

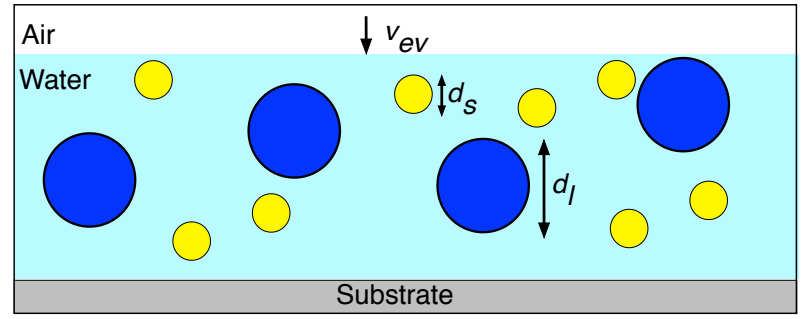

Figure 1. Sketch of a wet film containing a colloidal mixture of large particles with diameter $d_{l}$, and small particles with diameter $d_{s}$. The film is bounded at the bottom by a substrate and at the top by the air/water interface that falls with a velocity of $v_{e v}$.

Simulation: Figure 1 illustrates the system under consideration; a two-component colloidal suspension of large and small particles. The films are typically of the order of 

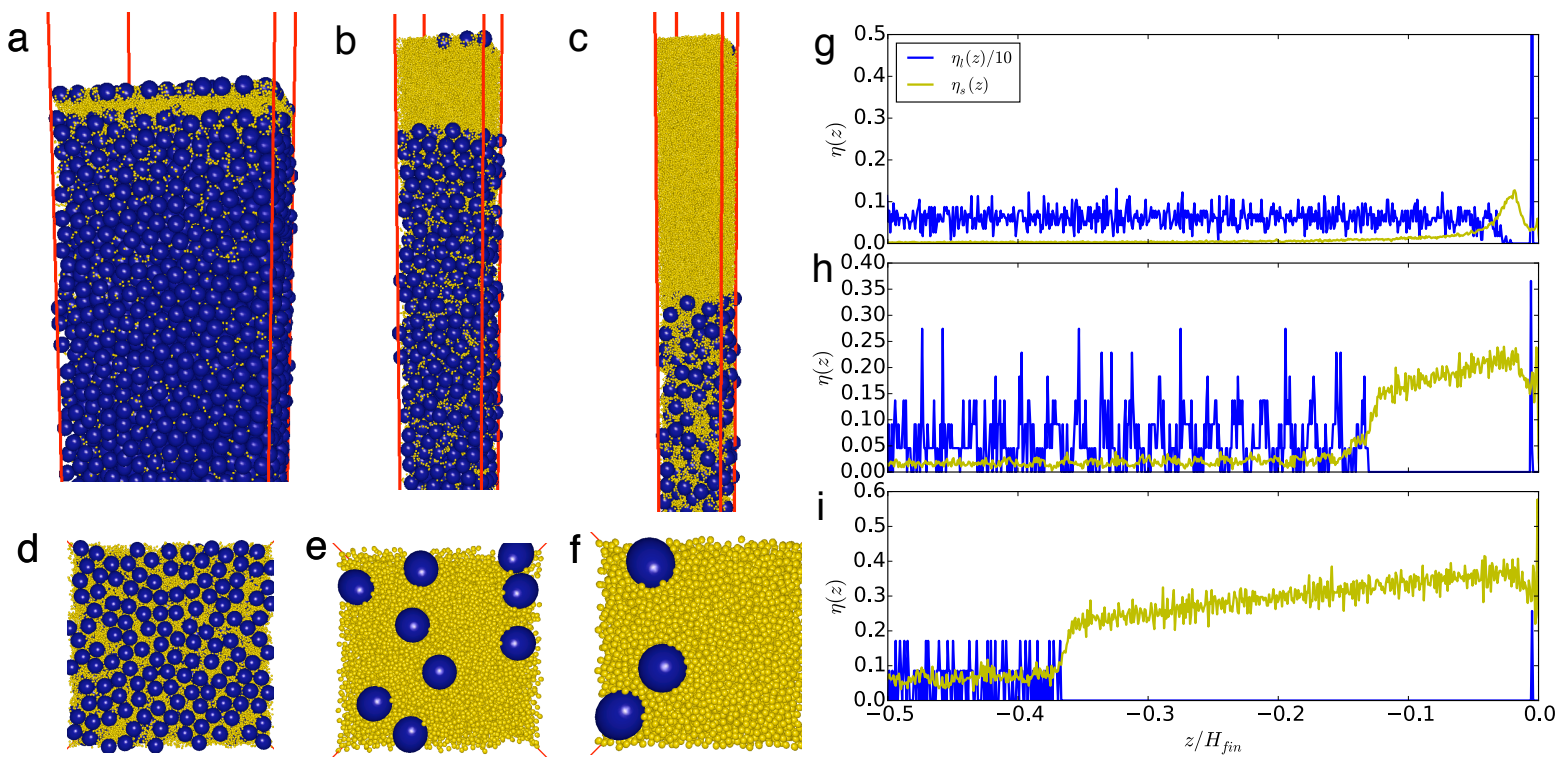

Figure 2. (a)-(f) Simulation snapshots of the top third of the simulation box, taken at the end of the run. The small particles are shown in yellow (light gray), the large particles in blue (dark gray), with size ratio $d_{l} / d_{s}$ of $7: 1$. In each case the system started at a total volume fraction $\eta_{0}=0.1$ and height $H=1500 d_{s}$ and was run until a final height of $H_{f i n}=303 d_{s}$. The snapshots in (a), (b) and (c) are for systems with increasing amounts of the small particles, the number ratios are $N_{r}=5,29$, and 151, respectively. The snapshots in (d), (e) and (f) are the top views of the systems in (a), (b) and (c), respectively. Due to the strong attraction between the surface and the large particles, even in (c) and (f), where stratification is strongest, we see some large particles trapped at the surface. Their presence does not affect stratification. (g)-(i) Volume fraction profiles of the small (yellow/light gray) and large (blue/dark gray) particles, for $N_{r}=5,29$ and 151, respectively. The bin width used in the profiles is $0.25 d_{s}$.

1000 particle diameters in height and macroscopic in the other two directions. At the top is the water/air interface and the substrate is at the bottom.

We carried out simulations on a binary mixture of spherical particles with diameters $d_{l}$ and $d_{s}$; the size ratio $d_{l} / d_{s}=7$. The interaction between particles is that of screened charged particles, which is modeled by a short range repulsive Yukawa interaction. In contrast to the simulation of [17], our model assumes stable particles over the time scale of the evaporation. The motion of the colloidal particles is simulated by Langevin dynamics [18], which includes Brownian diffusion but neglects hydrodynamic flow. The simulation box has dimensions $L_{x} \times L_{y} \times H$. To model a part of a large-area film that is far from any edges, we apply periodic boundary conditions in the $x$ - and $y$-directions. Evaporation occurs along the vertical, $z$ direction. We model the air/water interface by a harmonic potential for the particles, and evaporation is modeled by the potential's minimum moving downward at a constant velocity $v_{e v}=0.05 d_{s} / \tau_{B}$, where $\tau_{B}=d_{s}^{2} / D_{s}$ is the Brownian time, and $D_{s}$ is the Stokes-Einstein diffusion coefficient of the small particles. This gives a Péclet number for the small particles of 75 ; that for large particles is $d_{l} / d_{s}=7$ times larger. At the bottom we model the static substrate by the purely repulsive part of the Lennard-Jones interaction.
In all cases we start with $N_{s}$ small and $N_{l}$ large particles, such that the total initial volume fraction of the mixture $\eta_{0}=0.1$. However, we varied the ratio $N_{r}=N_{s} / N_{l}$. The system is equilibrated with a static top surface, and after equilibration for a time $100 \tau_{B}$, the downward movement of the model air/water interface begins. As the interface moves downward at a constant velocity $v_{e v}$, both small and large particles tend to accumulate below this moving interface. As the interface moves down, an accumulation region forms and grows with time. This is a region where the density is higher and there is a density gradient. A few large and small particles become trapped at the interface because of the effects of surface tension, while particles just below the surface diffuse normally. Inside the accumulation region where there is a density gradient, the large particles move away from the top region, creating a well-defined layer composed of only small particles [19]. The width of the layer depleted of large particles grows in time, as is shown in Fig. 1(f) of the Supplementary Material [20]. The layer continues to grow as long as the small particles can continuously filter through the large particles. This growth is hindered at higher volume fractions due to the slowing of the dynamics and the jamming of the small particles. The time for the segregation is larger than the time for evaporation for very high initial volume fractions. 
The simulations are run until the accumulation front reaches the bottom substrate. The film height is initially $H=1500 d_{s}$ and at the end of the simulation it is $H_{\text {fin }}<H$ [21]. We do not examine the later stages of film formation.

In Fig. 2(a)-(f) we show snapshots of the top portion of the simulation box, taken when the accumulation front has reached the bottom. We see in Fig. 2(c) that the small particles have formed a thick layer at the top that has excluded the larger particles. These larger particles have been pushed down into a separate layer, with smaller particles in the interstitial spaces between the larger particles. The thickness of the layer of small particles at the top of the film is lower when the number of small particles is reduced, as we can see in Fig. 2(a) and (b), but the layer is still present.

Note that equilibrium mixtures of large and small hard spheres with a size ratio of $7: 1$ are completely miscible in the fluid phase [22]. At high volume fraction there is a broad region of coexistence between a crystal of the large particles and a fluid composed of mainly the small particles. But in our simulations stratification pre-empts crystallization, and this stratificiation is inherently nonequilibrium in nature; it is not due to an underlying equilibrium phase separation.

The stratification effect is general and occurs at different size ratios and for a range of initial volume fractions. With high volume fractions, we find a smaller width of the layer of small particles due to jamming effects of the small particle [23].

Experiments: Such a striking segregation has not been reported before, therefore we carried out an experimental investigation to confirm the findings. Aqueous blends of colloidally stable acrylic copolymer particles $\left(d_{l} / d_{s}=\right.$ 7 ) at total volume fraction $\eta_{0}=0.1$ and varying number ratios were deposited on glass substrates with an initial wet thickness of approximately $\mathrm{H}=700 \mu \mathrm{m}$. We dried the samples at room temperature, leading to $\mathrm{Pe}_{\text {film }}=14$ and 100, for the small and large particles, respectively. After film formation, the final films were characterized by means of atomic force microscopy (AFM) and scanning confocal microscopy. In order to visualize the population distribution of large $(385 \mathrm{~nm})$ particles within the sample, they were labelled with a red fluorescent dye (Rhodamine B); the small $(55 \mathrm{~nm})$ particles were unlabelled [24].

Stratification is clearly seen in Fig. 3, although the layers are less distinct than in our simulations. Compare Fig. 2(g)-(i) to Fig. 3(g). In our experiments, we see stratification for the two mixtures with larger numbers of small particles, $N_{r}=200$ and 500 in the confocal images in Fig. 3(e) and (f), respectively. The distribution is uniform with $N_{r}=10$.

The surface coverage with small particles seen in the AFM images of Fig. 3(b) and (c) is also consistent with stratification. The AFM images show small particles at
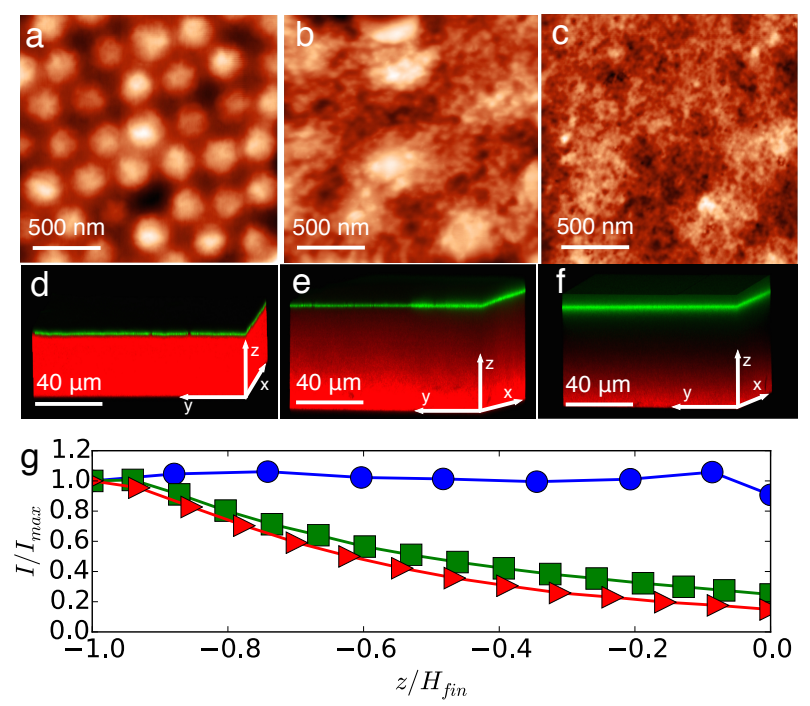

Figure 3. Experimental results for dried films formed of a binary mixture of colloidal particles of size ratio $7: 1$. (a)-(c) Height AFM images for films with number ratios $N_{r}=10$, 200 and 500, respectively. (d)-(f) Three dimensional confocal images of the films for $N_{r}=10,200$ and 500, respectively. The large particles are labelled with a red dye, while the small particles are unlabelled so the intensity of red indicates the concentration of the larger particles. To mark the position of the top surface, a drop of large $(750 \mathrm{~nm})$ green fluorescent particles was cast on the dry film. (g) Intensity of the red channel (large particles) as a distance from the top surface located at $z / H_{\text {fin }}=0$, for $N_{r}=10$ (blue circles), $N_{r}=200$ (green squares) and $N_{r}=500$ (red triangles).

the top surface for the number ratios $N_{r}=200$ and 500, but not for $N_{r}=10$. Thus for mixtures of particles of size ratio $7: 1$, we see stratification both in our computer simulations and experiments at sufficiently high number ratios.

Model: In order to understand the segregation of the large and small particles into layers, we develop a physical model. In the evaporating film, density and hence pressure gradients build up [25]. These gradients create forces that push particles of all sizes down the gradients, and away from the surface. Segregation results if these forces push the larger particles at faster speeds than the smaller ones. The speed of a particle of diameter $d$ depends on the balance between the force $f(d)$ on the particle, and the drag $\xi(d)$.

For simplicity, we will consider the case where a majority species of diameter $d_{m}$ dominates the osmotic pressure, $P$, but there is a trace amount of a species of a different diameter, $d_{t}$.

In the presence of a pressure gradient $\partial P / \partial z$, the difference in pressure between the top and bottom of a particle of diameter $d$ is $\approx d(\partial P / \partial z)$. So the net downward force on the particle $f(d) \approx d^{3}(\partial P / \partial z)$.

The friction coefficient of a particle of diameter $d$ is 
$\xi(\eta, d)=K(\eta, d) \xi_{0}[26,27]$. Here $\xi_{0}=3 \pi d \nu$ is the Stokes friction coefficient, with $\nu$ the viscosity of water. $K(\eta, d)$ is the sedimentation coefficient, defined as the ratio of the sedimentation velocity at volume fraction $\eta$ to that in its dilute limit.

At any point, the majority species will be pushed away from the interface at speed $v\left(d_{m}\right)=f\left(d_{m}\right) / \xi\left(d_{m}\right)$. Segregation of the tracer particles is determined by their velocity relative to that of the dominant species

$$
\Delta v\left(d_{t}\right)=v\left(d_{t}\right)-v\left(d_{m}\right)=v\left(d_{m}\right)\left(\frac{d_{t}^{2} K\left(\eta, d_{m}\right)}{d_{m}^{2} K\left(\eta, d_{t}\right)}-1\right)
$$

At low densities $K \simeq 1$, therefore the downward velocity of tracer particles relative to that of the majority species varies as $\left(d_{t} / d_{m}\right)^{2}-1$, i.e., it increases quadratically with the diameter of the tracer particles. Species larger than the majority species move down faster than the majority species, and segregation occurs with the larger particles at the bottom. On the other hand, species smaller than the majority species move down slower than the majority species, resulting in smaller particles accumulating at the top. The functional form of $K(\eta, d)$ has been the focus of many studies in theory, simulations and experiments [27-29] and does not depend on Péclet number [30]. For Brownian particles, the diameter dependence at high density is $K \simeq d$, which leads to a segregation velocity that scales as $\left(d_{t} / d_{m}\right)-1$, i.e., it would still lead to stratification. However, at high volume fraction the dynamics of the system slows considerably and the time scale for the segregation mechanism could become larger than the time for solvent evaporation [31].

Test of the model: Our simple theoretical model makes a striking prediction: larger particles move down relative to the majority species, while smaller ones move up. This can be independently verified by simulating mixtures with a majority species plus both smaller and larger particles. Therefore, we simulated a ternary mixture of particles: a majority species with diameter $d_{m}$, and two minority components with size ratios $d_{s} / d_{m}=0.8$ and $d_{b} / d_{m}=1.2$. The initial average volume fractions are $\eta_{m}=0.05, \eta_{s}=0.003, \eta_{b}=0.0052$ and the evaporation velocity is $v_{e v}=0.1 d_{m} / \tau_{B}$.

The results for the ternary mixture are shown in Fig. 4. In Fig. 4(a) we see that the moving surface has created gradients in the density and hence in the pressure of the majority species (shown in yellow/light gray), of width $\approx 150 d_{m}$. The gradient of the osmotic pressure is plotted in Fig. 4(b). The dominant force is due to the osmotic pressure gradient of the majority species.

The larger species are on average farther from the top surface than the majority species. Note the maximum in their density (shown in blue/dark gray) around $100 d_{m}$ below the surface. By contrast, the smaller particles (shown in black) are accumulating near the top surface. As predicted, at our large film formation Péclet numbers, mixtures of particles of different sizes are unstable with respect to stratification into layers, with the smallest particles at the top, and the largest at the bottom.

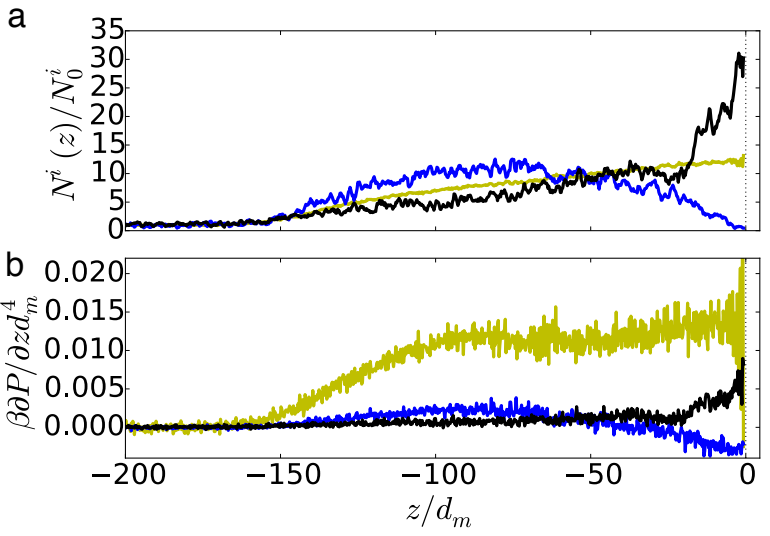

Figure 4. Density and pressure gradient profiles in the drying film at time $\mathrm{t}=4350 \tau_{B}$ for ternary mixtures. The top surface is at $z / d_{m}=0$. The yellow (light gray) curves represent the majority species, the blue (dark gray) curves represent the larger species, $d_{b} / d_{m}=1.2$, and the black curves represent the smaller species, $d_{s} / d_{m}=0.8$. (a) Densities of particles as a function of the distance from the interface, plotted as $N^{i}(z) / N_{0}^{i}$, where $N^{i}(z)$ is the density of species $i=m, s, b$ and $N_{0}^{i}$ is the initial number density. (b) Vertical gradients of the osmotic pressure as a function of the distance from the top interface. The component of the dominant species makes the greatest pressure contribution. The vertical dotted lines indicate the position of the top interface.

In our simulations we neglected any effect due to hydrodynamic flow of the solvent. Modeling flow for our systems of many thousands of particles is not computationally feasible. Flow is present in the experiments of course. When the volume fraction of the drying suspension is changing, there will be relative motion of the particles and water, which will generate forces on the particles acting toward the surface. On one hand, these forces will push larger particles toward the surface and counteract the segregation of small particles. On the other hand, the majority species will be pushed toward the surface and create larger osmotic pressure gradients that will enhance segregation. We cannot calculate these forces, but we note that the effect we describe here is very robust in the simulations. Furthermore, we see the effect in experiments where there is hydrodynamic flow of water. Hence, we believe that stratification does occur in the presence of forces due to hydrodynamic flow.

Discussion and Conclusion: In both computer simulations and experiments on drying colloidal mixtures, we found stratification. The smaller particles excluded the larger particles and formed a layer at the top of the drying film. This is a purely out-of-equilibrium effect; it is driven by the moving interface. The moving interface causes a density, and hence a pressure, gradient in 
the drying film, and this pressure gradient pushes larger particles away from the moving interface faster than it pushes smaller particles. We developed a physical model for this process, and the model correctly predicted the behavior of both small and large particles.

Diverse technologies, including inkjet printing [32], coatings on pharmaceutical tablets [33, 34], agricultural treatments on crops [35-37], synthetic latex paints, adhesives [3], and cosmetics (such as sun screen [38]), rely on films derived from mixtures of colloidal particles. Our discovered mechanism will be useful whenever the properties of the top and the bottom of a coating need to be controlled independently via a one-step deposition process.

We acknowledge funding from the European Union Seventh Framework Programme BARRIER-PLUS project (FP7-SME-2012-2, no. 304758).

* andrea.fortini@me.com

$\dagger$ r.sear@surrey.ac.uk

[1] A. F. Routh, Rep. Prog. Phys. 76, 1 (2013).

[2] R. Brown, Phil. Mag. 4, 161 (1828).

[3] J. L. Keddie and A. F. Routh, Fundamentals of Latex Film Formation, Processes and Properties (Springer, 2010).

[4] A. F. Routh and W. B. Zimmerman, Chem. Eng. Sci. 59, 2961 (2004).

[5] R. E. Trueman, E. L. Domingues, S. N. Emmett, M. W. Murray, and A. F. Routh, J. Colloid Interface Sci. 377, 207 (2012).

[6] J.-P. Gorce, D. Bovey, P. J. McDonald, P. Palasz, D. Taylor, and J. L. Keddie, Eur. Phys. J. E 8, 421 (2002).

[7] P. Ekanayake, P. J. McDonald, and J. L. Keddie, Eur. Phys. J. ST 166, 21 (2009).

[8] C. M. Cardinal, Y. D. Jung, K. H. Ahn, and L. F. Francis, AIChE J. 56, 2769 (2010).

[9] Y. Reyes, J. Campos-Terán, F. Vázquez, and Y. Duda, Model. Simul. Mater. Sci. Eng. 15, 355 (2007).

[10] S. Cheng and G. S. Grest, J. Chem. Phys. 138, 064701 (2013).

[11] E. Rabani, D. R. Reichman, P. L. Geissler, and L. E. Brus, Nature 426, 271 (2003).

[12] J. Zhang, Y. Li, X. Zhang, and B. Yang, Adv. Mater. 22, 4249 (2010).

[13] H. Luo, C. M. Cardinal, L. E. Scriven, and L. F. Francis, Langmuir 24, 5552 (2008).
[14] A. K. Atmuri, S. R. Bhatia, and A. F. Routh, Langmuir 28, 2652 (2012).

[15] I. Nikiforow, J. Adams, A. M. König, A. Langhoff, K. Pohl, A. Turshatov, and D. Johannsmann, Langmuir 26, 13162 (2010).

[16] M. Schröter, S. Ulrich, J. Kreft, J. Swift, and H. Swinney, Phys. Rev. E 74, 011307 (2006).

[17] Q. Liao, L. Chen, X. Qu, and X. Jin, J. Colloid Interface Sci. 227, 84 (2000).

[18] S. Plimpton, J. Comp. Phys. 117, 1 (1995).

[19] See Supplemental Material at [URL] for the movie EvaporationNr151.avi showing this effect, and Fig 1 of supplementary.pdf at [URL].

[20] See Supplemental Material at [URL] for a sequence of density profiles at different times.

[21] See Supplemental Material at [URL] for simulation details.

[22] M. Dijkstra, R. van Roij, and R. Evans, Phys. Rev. E 59, 5744 (1999).

[23] See Supplemental Material at [URL] for snapshots of the final configurations at different volume fractions.

[24] See Supplemental Material at [URL] for details of experiments and particle synthesis.

[25] See Fig. 1 of Supplemental Material at [URL].

[26] C. Van den Broeck, F. Lostak, and H. Lekkerkerker, J. Chem. Phys. 74, 2006 (1981).

[27] W. B. Russel, D. A. Saville, and W. R. Schowalter, Colloidal Dispersions (Cambridge University Press, 1992).

[28] H. Hayakawa and K. Ichiki, Phys. Rev. E 51, R3815 (1995).

[29] W. R. Bowen, Y. Liang, and P. M. Williams, Chem. Eng. Sci. 55, 2359 (2000).

[30] J. T. Padding and A. A. Louis, Phys. Rev. Lett. 93, 220601 (2004).

[31] S. Peppin and J. Elliott, Journal of Fluid Mechanics 554, 147 (2006).

[32] E. Tekin, P. J. Smith, and U. S. Schubert, Soft Matter 4, 703 (2008).

[33] S. Obara and J. W. McGinity, International journal of pharmaceutics 126, 1 (1995).

[34] F. Lecomte, J. Siepmann, M. Walther, R. J. MacRae, and R. Bodmeier, Pharmaceutical Research 21, 882 (2004).

[35] M. A. Faers and R. Pontzen, Pest management science 64, 820 (2008).

[36] P. Taylor, Current Opinion in Colloid \& Interface Science 16, 326 (2011).

[37] M. A. Hampton, T. A. H. Nguyen, A. V. Nguyen, Z. P. $\mathrm{Xu}, \mathrm{L}$. Huang, and V. Rudolph, Journal Of Colloid And Interface Science 377, 456 (2012).

[38] S. A. Wissing and R. H. Müller, International journal of cosmetic science 23, 233 (2001). 